Hechos Microbiol. 2019;10(1-2):23-29.

DOI: 10.17533/udea.hm.v10n1a02

(C) 2019 por la Universidad de Antioquia

http://www.udea.edu.co/hm

\title{
Gestión del reporte de valores críticos en pacientes ambulatorios de un laboratorio clínico
}

\author{
Report management of critical values in ambulatory patients of a clinical laboratory
}

Catalina Madrid Osorio ${ }^{*}$, Johana Hernández Vargas", Melisa Villa*, Mary Alejandra Restrepo Lozada

\section{RESUMEN}

INTRODUCCIÓN: el laboratorio clínico ha pasado de desempeñar un papel pasivo centrado en la calidad analítica de sus resultados, a un papel activo y protagónico basado en la toma de decisiones clínicas y la seguridad del paciente. En este estudio se midieron los tiempos de reporte de resultados críticos en pacientes ambulatorios con el fin de determinar la adherencia al protocolo por parte del personal asistencial del Laboratorio Médico Echavarría.

MÉTODOS: se realizó un análisis retrospectivo de los reportes de resultados críticos emitidos por el Laboratorio Médico Echavarría, entre julio de 2017 y junio de 2018. Para el análisis se tuvo en cuenta una muestra de 269 resultados críticos en pacientes ambulatorios particulares.

RESULTADOS: se identificó que $29(10,7 \%)$ pertenecían a la sección de coagulación, 174 (64,6 \%) a la sección de hematología, $65(24,1 \%)$ a la sección de química y hormonas y $1(0,3 \%)$ a la sección de inmunología básica. Se encontró un tiempo promedio de reporte de 33,4 minutos en todas las secciones.

CONCLUSIONES: cada uno de los laboratorios, debe establecer, controlar y documentar con base en su lista de valores críticos y la población atendida, el tiempo de demora en la comunicación de los resultados críticos; su disminución debe hacer parte de la mejora continua en el sistema de gestión de calidad al que se adhiera.

PALABRAS CLAVE: evento adverso, incidente, notificación, paciente ambulatorio, seguridad del paciente, valores críticos.

* Laboratorio Médico Echavarría, Medellín, Colombia.

† Contacto: servicioalcliente2@labechavarria.com

Recepción: 27-05-2019. Aceptación: 05-11-2019

Cómo citar este artículo: Madrid-Osorio C, Hernández-Vargas J, Restrepo-Lozada MA.Gestión del reporte de valores críticos en pacientes ambulatorios de un laboratorio clínico. Hechos Microbiol. 2019;10(1-2):23-29. DOI: 10.17533/udea.hm.v10n1a02 


\section{ABSTRACT}

INTRODUCTION: The clinical laboratory has gone from playing a passive role, focused on the analytical quality of its results, to an active and leading role, based on clinical decisions and patient safety. In this study, we measured the reporting times of critical results in outpatients in order to determine the adherence to protocol by the staff of the Laboratorio Medico Echavarria.

METHODS: A retrospective analysis of the critical results released by the Laboratorio Médico Echavarría was conducted between July 2017 and June 2018. In total, a sample of 269 critical results for private outpatients was taken into account for the analysis.

RESULTS: It was identified that $29(10.7 \%)$ belonged to the coagulation section, $174(64.6 \%)$ to the hematology section, $65(24.1 \%)$ to the chemistry and hormones section and $1(0.3 \%)$ to the basic immunology section. An average report time of 33.4 minutes was found in all sections.

CONCLUSIONS: Each laboratory must establish, control and document the delay in communicating the critical results, based on its list of critical values and the population served; its reduction must be part of the continuous improvement in the quality management system that is followed.

KEY WORDS: ambulatory patient, adverse event, critical values, incident, notification, patient safety.

\section{INTRODUCCIÓN}

El laboratorio clínico es un área de especial importancia en la atención en salud, se calcula que el $80,0 \%$ de las decisiones médicas están sustentadas en los resultados de las pruebas biológicas. ${ }^{1}$ Parte de estas decisiones se derivan del reporte de los valores críticos a cargo del laboratorio, para lo cual se requieren mecanismos de comunicación efectiva, oportuna, correcta, inequívoca y comprensible para cada uno de los receptores de la información. ${ }^{2}$

En 1972, Lundberg, acuñó el término de "valores críticos" para aquellos indicadores de un estado fisiopatológico alejado de la normalidad que puede poner en peligro la vida del paciente si no se actúa rápidamente, y para el que existe tratamiento. ${ }^{3}$ La correcta gestión de los resultados o valores críticos conlleva a la toma de decisiones asertivas y oportunas en salud, permitiendo un manejo adecuado de los pacientes y minimizando el riesgo de presentar eventos adversos e incidentes durante la atención en salud. ${ }^{4,5}$

Para esta gestión es indispensable contar con un protocolo de manejo de resultados críticos. Sin embargo, actualmente no existe una política de reporte de resultados críticos universalmente aceptada o consensuada con la comunidad de usuarios; por lo cual, cada laboratorio clínico debe establecer la propia, que incluya los procedimientos asociados al reporte, los mecanismos de comunicación y el listado de los resultados críticos; los cuales deben resultar como consenso por los profesionales del laboratorio, personal médico y otros servicios involucrados en la atención de los pacientes conforme a las necesidades de la institución, las características de la población atendida y la prevalencia de las enfermedades atendidas, entre otros.1 Por ejemplo, el Colegio Americano de Patólogos (CAP) propuso, en el año 2002, una lista de resultados críticos que actualmente sirve como punto de partida para los laboratorios clínicos. ${ }^{6}$

En el Laboratorio Médico Echavarría (LME) se ha establecido un protocolo para la gestión del reporte de resultados críticos, en el cual se tiene definido el listado de los valores críticos, el análisis y la comunicación de los mismos.

Por lo tanto, con este estudio se pretende presentar el análisis del comportamiento de la gestión de los resultados críticos, en el ámbito de un laboratorio clínico privado y en la atención de pacientes ambulatorios.

\section{MATERIALES Y MÉTODOS}

Se realizó un análisis retrospectivo de los reportes de resultados críticos emitidos por el LME a pacientes particulares que solicitaron realizarse análisis paraclínicos, entre julio de 2017 y junio de 2018. Las muestras se obtuvieron de acuerdo a los protocolos definidos por el laboratorio para la toma, identificación y transporte. Los valores críticos fueron determinados por el LME de acuerdo a las guías internacionales del del CAP, la Federación Internacional de Química Clínica (IFCC, por las siglas en inglés International 
Federation of Clinical Chemistry), y del Comite Técnico Operativo al interior del LME (Tabla 1). En ninguno de los casos se tuvo en cuenta el diagnóstico del paciente o su estado fisiopatológico, dado que, al ser ambulatorios, no se contaba con la historia clínica de los mismos, por lo tanto cualquier episodio catalogado como crítico fue incluido.

Tabla 1. Valores críticos adaptados del Laboratorio Médico Echavarría

\begin{tabular}{|c|c|c|c|c|c|}
\hline \multirow{2}{*}{ Prueba / Unidades } & \multicolumn{2}{|c|}{ Adultos } & \multicolumn{2}{|c|}{ Pediátricos } & \multirow{2}{*}{ Referencia } \\
\hline & Inferior & Superior & Inferior & Superior & \\
\hline \multicolumn{6}{|l|}{ Química clínica } \\
\hline Ácido úrico / mg/dL & NA & $\geq 13,0$ & NA & $\geq 13,0$ & IFCC \\
\hline Bilirrubina / mg/dL & NA & $\geq 15$ & NA & $\geq 14$ & IFCC \\
\hline Calcio lónico / mmol/L & $\leq 0,8$ & $\geq 1,6$ & $\leq 0,8$ & $\geq 1,6$ & IFCC \\
\hline Calcio / mg/dL & $\leq 6,4$ & $\geq 12,8$ & $\leq 6,4$ & $\geq 12,8$ & CAP \\
\hline $\mathrm{CK} / \mathrm{U} / \mathrm{L}$ & NA & $\geq 1000$ & NA & $\geq 1000$ & IFCC \\
\hline Cloro / mmol/L & $\leq 75$ & $\geq 125$ & $\leq 75$ & $\geq 125$ & IFCC \\
\hline Creatinina / mg/dL & NA & $\geq 7,4$ & NA & $\geq 3,8$ & IFCC \\
\hline Fósforo / mg/dL & $\leq 1$ & $\geq 9$ & $\leq 1$ & $\geq 9$ & IFCC \\
\hline Glucosa / mg/dL & $\leq 45$ & $\geq 450$ & $\leq 30$ & $\geq 325$ & IFCC \\
\hline Lipasa / U/L & NA & $\geq 700$ & NA & $\geq 700$ & IFCC \\
\hline Litio / mEq/L & NA & $\geq 2$ & NA & $\geq 2$ & CLR \\
\hline Magnesio / mg/dL & $\leq 0,88$ & $\geq 4,07$ & $\leq 0,88$ & $\geq 4,07$ & CAP \\
\hline Nitrógeno Ureico / mg/dL & NA & $\geq 100$ & NA & $\geq 55$ & IFCC \\
\hline Osmolalidad / mOsm/Kg & $\leq 240$ & $\geq 330$ & $\leq 240$ & $\geq 330$ & IFCC \\
\hline $\mathrm{pCO}_{2} / \mathrm{mm} \mathrm{Hg}$ & $\leq 19$ & $\geq 67$ & $\leq 19$ & $\geq 67$ & IFCC \\
\hline $\mathrm{PCR} / \mathrm{mg} / \mathrm{L}$ & NA & NA & NA & $\mathrm{RN} \geq 5,0$ & IFCC \\
\hline $\mathrm{pH}$ & $\leq 7,2$ & $\geq 7,6$ & $\leq 7,2$ & $\geq 7,6$ & IFCC \\
\hline $\mathrm{pO}_{2} / \mathrm{mm} \mathrm{Hg}$ & $\leq 43$ & NA & $\mathrm{RN} \leq 37$ & NA & IFCC \\
\hline Potasio / mEq / L & $\leq 2,5$ & $\geq 6$ & $\leq 2,5$ & $\geq 6$ & CAP \\
\hline Sodio / mEq /L & $\leq 120$ & $\geq 160$ & $\leq 120$ & $\geq 160$ & IFCC \\
\hline Transaminasas / U/L & NA & $\geq 1000$ & NA & $\geq 1000$ & IFCC \\
\hline Troponina / mg/L & NA & $\geq 0,1$ & NA & $\geq 0,1$ & IFCC \\
\hline \multicolumn{6}{|l|}{ Hormonas } \\
\hline $\mathrm{T} 4 \mathrm{~L} / \mathrm{ng} / \mathrm{dL}$ & NA & $\geq 3,5$ & NA & $\geq 3,5$ & IFCC \\
\hline \multicolumn{6}{|l|}{ Coagulación } \\
\hline Dímero D & NA & 0,4 & NA & 0,4 & IFCC \\
\hline Fibrinógeno / mg/dL & $\leq 70$ & $\geq 1000$ & $\leq 70$ & $\geq 1000$ & IFCC \\
\hline $\mathrm{TP} / \mathrm{s}$ & NA & INR $\geq 4,5$ & NA & INR $\geq 4,5$ & Campuzano $^{7}$ \\
\hline TPT / s & NA & $\geq 85$ & NA & $\geq 75$ & Campuzano $^{7}$ \\
\hline \multicolumn{6}{|l|}{ Hematología } \\
\hline \multirow{2}{*}{ Plaquetas / mm3 } & $\leq 20000$ & $\geq 1000000$ & $\leq 20000$ & $\geq 1000000$ & IFCC \\
\hline & & & $\mathrm{RN} \leq 100000$ & & LME \\
\hline Hemoglobina / g/dL & $\leq 7,0$ & $\geq 19,9$ & $\leq 8,5$ & $\geq 23,0$ & CAP \\
\hline
\end{tabular}




\begin{tabular}{|c|c|c|c|c|c|}
\hline \multirow{2}{*}{ Prueba / Unidades } & \multicolumn{2}{|c|}{ Adultos } & \multicolumn{2}{|c|}{ Pediátricos } & \multirow{2}{*}{ Referencia } \\
\hline & Inferior & Superior & Inferior & Superior & \\
\hline \multirow{2}{*}{ Leucocitos / mm3 } & $\leq 2$ & $\geq 50$ & $\leq 2$ & $\geq 50$ & IFCC \\
\hline & & & $\mathrm{RN} \leq 5$ & $\mathrm{RN} \geq 25$ & LME \\
\hline Hematocrito / \% & $\leq 18$ & $\geq 61$ & $\mathrm{RN} \leq 33$ & $\mathrm{RN} \geq 71$ & IFCC \\
\hline Neutrófilos / mm3 & $\leq 0,5$ & NA & $\leq 0,5$ & NA & IFCC \\
\hline \multicolumn{6}{|l|}{ Microbiología } \\
\hline Hemocultivo & NA & Positivo & NA & Positivo & CAP \\
\hline Gram (muestra estéril) & NA & Positivo & NA & Positivo & CAP \\
\hline \multicolumn{6}{|l|}{ Inmunologia Básica } \\
\hline Dengue Anticuerpos IGM & NA & Positivo & NA & Positivo & LME \\
\hline
\end{tabular}

En la Figura 1 se describe el algoritmo utilizado en el LME para el correcto reporte de resultados críticos.

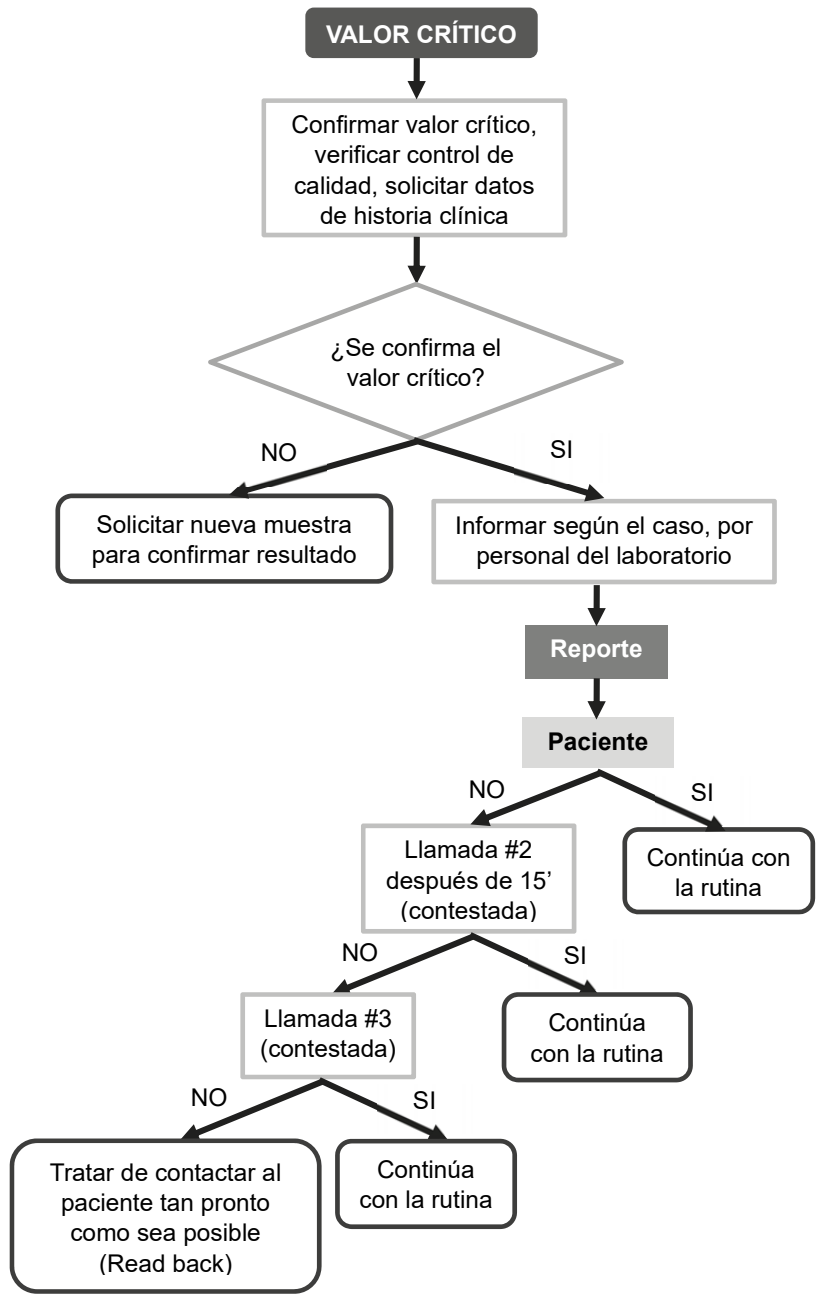

Figura 1. Flujograma de reporte de resultados críticos en el LME
Se elaboró una base de datos en formato Microsoft Office Excel 2010, en la cual se consignó la información correspondiente a: número de solicitud, fecha, parámetro, sección, resultado crítico, hora de validación del resultado crítico, hora de reporte del resultado crítico, tiempo de reporte, responsable del reporte, horas de llamadas, read-back y motivo de no informe (según el caso). Se excluyeron aquellos reportes que no tuvieran toda la información completa.

Se calcularon las frecuencias absolutas y relativas, para la variable parámetro con resultado y tiempo de notificación del resultado, el cual fue computado a partir de la hora de validación y la hora en la que se notificó el dato.

En relación al cumplimiento del reporte por medio de los profesionales de laboratorio, se tuvo en cuenta la meta del indicador de reporte en menos de tres horas por ser un laboratorio ambulatorio, por medio de comunicación verbal y read back (estrategia basada en el entendimiento del receptor del mensaje). ${ }^{4}$

La medición se realizó basada en el Sistema de Información de Laboratorio (LIS Athenea), el cual cuenta con trazabilidad de la información en todo el proceso de la atención del paciente.

\section{RESULTADOS}

Durante el periodo de estudio (julio de 2017 a junio de 2018) fueron identificados 159.780 reportes de pacientes en estado ambulatorio, de los cuales fueron identificados 269 reportes de resultados críticos. De los 269 resultados críticos, se identificó que 29 (10,7\%) 
pertenecían a la sección de coagulación (C), 174 $(64,6 \%)$ a la sección de hematología $(\mathrm{H}), 65(24,1 \%)$ a la sección de química y hormonas $(\mathrm{QH})$ y $1(0,3 \%)$ a la sección de inmunología básica (IB), tal como se muestra en la Tabla 2. Identificando que la sección de hematología es donde se reportan la mayor cantidad de pruebas críticas en pacientes ambulatorios.

Tabla 2. Seguimiento del reporte de los resultados críticos por sección

\begin{tabular}{|l|l|c|c|c|c|c|}
\hline \multicolumn{1}{|c|}{ Mes } & Año & Coagulación & Hematología & Inmunología Básica & Química y Hormonas & Total \\
\hline Julio & 2017 & 2 & 11 & 0 & 3 & 16 \\
\hline Agosto & 2017 & 1 & 12 & 0 & 9 & 22 \\
\hline Septiembre & 2017 & 1 & 8 & 0 & 9 & 18 \\
\hline Octubre & 2017 & 3 & 17 & 0 & 6 & 26 \\
\hline Noviembre & 2017 & 5 & 13 & 1 & 3 & 22 \\
\hline Diciembre & 2017 & 2 & 17 & 0 & 5 & 24 \\
\hline Enero & 2018 & 0 & 17 & 0 & 4 & 21 \\
\hline Febrero & 2018 & 1 & 12 & 0 & 4 & 17 \\
\hline Marzo & 2018 & 1 & 13 & 0 & 4 & 18 \\
\hline Abril & 2018 & 4 & 8 & 0 & 6 & 18 \\
\hline Mayo & 2018 & 4 & 23 & 0 & 5 & 32 \\
\hline Junio & 2018 & 5 & 23 & 0 & 7 & 35 \\
\hline Total & & $29(10,7 \%)$ & $174(64,6 \%)$ & $1(0,3 \%)$ & $65(241 \%)$ & $269(100 \%)$ \\
\hline
\end{tabular}

Se revisaron los resultados críticos por analito y se identificó que el más frecuente en pacientes ambulatorios fue el recuento de glóbulos blancos con un $24,2 \%$, seguido de hemoglobina, recuento de plaquetas, INR y bilirrubina total, con unos porcentajes de $22,7 \%, 14,9 \%, 8,9 \%$ y $7,4 \%$ respectivamente, lo que evidenció una mayor frecuencia de reportes críticos en el área de hematología.

Se verificó el porcentaje de adherencia en el reporte de los resultados críticos y el tiempo promedio tomado para este, con una media de 0,41 minutos (DS+/- 0,6) con un rango mínimo de 0 y máximo de 3 minutos y un nivel de confianza de 0,08 . La sección de coagulación tuvo un tiempo promedio del reporte oportuno de 44,33 minutos logrando un $62 \%$ de cumplimento; así mismo para las demás secciones, hematología con 37,05 y $77 \%$, inmunología básica con 37,00 y $100 \%$ y química y hormonas con 15,45 y $72,3 \%$.

Adicionalmente, se determinaron los porcentajes de incumplimiento de los tiempos de reporte, con el fin de establecer cuales se presentaron por falta de adherencia al protocolo y cuales por causas externas como lo indica el algoritmo, (se realizan tres llamadas y el paciente no contesta, datos errados y línea ocupada) evidenciando que el mayor número de incumpliendo por falta de adherencia al protocolo se presentó en el área de química y hormonas con un $77,7 \%$, coagulación con un $72,7 \%$, y hematología con un $27,5 \%$, resultados que fueron calculados teniendo como el $100 \%$ el número total de incumplimientos (Tabla 3).

Tabla 3. Motivo de incumplimiento del tiempo de reporte del resultado crítico

\begin{tabular}{|l|c|c|c|}
\hline \multicolumn{1}{|c|}{ Sección } & Número de incumplimientos & $\begin{array}{c}\text { Número de incumplimientos } \\
\text { por protocolo }\end{array}$ & $\begin{array}{c}\text { Número de incumplimientos } \\
\text { por causa externa }\end{array}$ \\
\hline Coagulación & 11 & 8 & 3 \\
\hline Hematología & 40 & 11 & 29 \\
\hline Química y Hormonas & 18 & 14 & 4 \\
\hline Total & $69(100 \%)$ & $33(47,8 \%)$ & $36(52,2 \%)$ \\
\hline
\end{tabular}


Con relación al total de incumplimientos por adherencia al protocolo presentados en el estudio, se obtuvieron $33(47,8 \%)$ casos, de los cuales $14(20,3 \%)$ finalmente no lograron ser reportados debido a causas externas y de los $19(27,5 \%)$ restantes, se obtuvo una media de 17,6 minutos (DS $\pm 5,7$ ), con un mínimo de 3,27 horas y un máximo de 24,5 horas y un nivel de confianza de 2,77 .

\section{DISCUSIÓN}

Teniendo en cuenta los analitos reportados como críticos en pacientes ambulatorios, el área que tuvo una mayor frecuencia de resultados críticos en el LME de la ciudad de Medellín fue hematología, en su mayoría por recuento bajos de glóbulos blancos; seguido del área de química y hormonas principalmente por el analito bilirrubina total.

Cabe resaltar que en estudios previos se ha sugerido que el reporte de resultados críticos se puede realizar en menor tiempo, entre 8 y 15 minutos. ${ }^{8} 9$ Esto evidencia que todavía se presenta una variación considerable en las prácticas y los intervalos de tiempo entre los laboratorios clínicos.

Los resultados críticos son claramente importantes en el aseguramiento de la calidad y la seguridad del paciente en todo tipo de casos (pacientes hospitalizados, pacientes en consulta y pacientes en urgencias, entre otros).

Es menester de los laboratorios clínicos, conocer y darles la importancia a los pacientes ambulatorios con valores críticos en diferentes analitos, así esta población represente un bajo porcentaje; primordialmente, se debe asegurar la salud y el bienestar de cada uno de ellos independientemente de las condiciones en las cuales acuden al laboratorio.

Cada uno de los laboratorios debe establecer, controlar y documentar con base en su lista de valores críticos y la población atendida, el tiempo de demora en la comunicación de los resultados críticos; su disminución debe hacer parte de la mejora continua en el sistema de gestión de calidad al que se adhiera. ${ }^{10,11}$ La comunicación verbal de los resultados críticos ha sido el estándar para la notificación de los mismos, sin embargo y gracias a los adelantos tecnológicos se han aceptado otras formas de reporte como los correos electrónicos. ${ }^{12}$
Establecer el protocolo para el reporte de resultados críticos ha ayudado a la estandarización de procesos dentro del laboratorio clínico, lo que proporciona un mayor cumplimiento en el sistema de gestión de la calidad de cada entidad. ${ }^{13}$

\section{CONCLUSIONES}

En este estudio se encontró que los analitos con un mayor número de reportes de resultados críticos en pacientes ambulatorios fueron glóbulos blancos, hemoglobina, recuento de plaquetas, INR y bilirrubina total. Además, se obtuvo un tiempo promedio de reporte de 33,4 minutos, en relación con las 3 horas definidas como meta. Para aquellos resultados que no fueron reportados oportunamente, las causas más frecuentes fueron la no respuesta del paciente luego de la tercer llamada, línea ocupada y datos errados de contacto. Esto evidencia una buena adherencia al protocolo para la gestión del reporte de resultados críticos en pacientes ambulatorios en el laboratorio, contribuyendo así a la toma de decisiones médicas asertivas y oportunas que favorecen el correcto manejo clínico de los pacientes, la oportunidad en el tratamiento, prevención de complicaciones, entre otras acciones que mejoren la seguridad de los pacientes.

Es importante generar estrategias constantes para garantizar un reporte oportuno de los resultados críticos empleando herramientas tecnológicas que permitan sistematizar la comunicación del dato a los clínicos; el Laboratorio Médico Echavarría en busca de la mejora continua, cuenta con un Clinical Decisión Support (CDS) el cual se encuentra en prueba para que el reporte de los resultados en rangos críticos se realice inmediatamente se emita el resultado, desde el sistema a los correos de los médicos tratantes o entidades de salud.

\section{AGRADECIMIENTOS}

Los autores agradecen al personal del Laboratorio Médico Echavarría, tanto de las secciones de laboratorio como del área administrativa, que participan activamente en la gestión del reporte de los resultados críticos. 


\section{CONFLICTO DE INTERÉS}

Los autores declaran no tener ningún conflicto de interés para la ejecución de este proyecto de investigación y la divulgación de sus resultados.

\section{REFERENCIAS}

1. Pérez-Valero V. El Laboratorio Clínico en el Sistema Asistencial. Semergen 2011;37(3):111-112.

2. ISO. Norma ISO 15.189:2014 (apartado 5.8) para Laboratorios Clínicos.

3. Lundberg GD. When to panic over abnormal values. MLO Med Lab Observ. 1972;4:47-54.

4. Guzmán A, Lagos M. Valores de Alerta en el Laboratorio Clínico y su Aporte al Cuidado y Seguridad del Paciente. Rev Med Chile. 2009;137:582-584

5. Joint Commission on Accreditation of Healthcare Organizations (JCAHO) National Patient Safety Goals. 2016. Disponible en: https://www.jointcommission.org/standards_information/jcfaqdetails.aspx ?StandardsFaqld=1050\&Programld=46
6. Cap.org. College of American Pathologist (CAP). Commission on Laboratory Accreditation. Disponible en: https://www.cap.org/

7. Campuzano G. Valores críticos: de la teoría a la práctica. Med y Lab. 2011; 7(17):331-50.

8. Wagar EA. Critical Values Reporting: An overview. (Com). American Society for Critical Pathology (ASCP) 2008 Annual Meeting October 16-19, 2008, Baltimore, Maryland.

9. Howanitz PJ, Steindel SJ, Heard NV. Laboratory critical valuespolicies and procedures: A College of American Pathologists Q-Probes study in 623 institutions. Arch Pathol Lab Med.2002;126:663-9.

10. Emancipator K. Critical values: ASCP practice parameter. Am J Clin Pathol. 1997; 108:147-53.

11. Tillman J, Barth JH. A survey of laboratory "critical (alert) limits" in the UK. Ann Clin Biochem. 2003;40:181-4.

12. Lippi G, Mattiuzzi C. Critical laboratory values communication: summary recommendations from available guidelines. Ann Transl Med. 2016;4(20):400.

13. Parl F, O'Leary M, Kaiser A, Paulett J, Statnikova, Shultz E. Implementation of a Closed-Loop Reporting System for Critical Values and Clinical Communication in Compliance with Goals of The Joint Commission. Rev Clinical Chemistry. 2010;56(3):417-423. 